\title{
Lagrangian Reduction, the Euler-Poincaré Equations, and Semidirect Products
}

\author{
Hernán Cendra \\ Universidad Nacional del Sur \\ 8000 Bahia Blanca, Argentina \\ uscendra@criba.edu.ar \\ Darryl D. Holm \\ Theoretical Division and Center for Nonlinear Studies \\ Los Alamos National Laboratory, MS B284 \\ Los Alamos, NM 87545 \\ dholm@lanl.gov \\ Jerrold E. Marsden* \\ Control and Dynamical Systems \\ California Institute of Technology 107-81 \\ Pasadena, CA 91125 \\ marsden@cds.caltech.edu \\ Tudor S. Ratiu ${ }^{\dagger}$ \\ Department of Mathematics \\ University of California, Santa Cruz, CA 95064 \\ ratiu@math.ucsc.edu \\ February 1997; this version, October 8, 1997 \\ Amer. Math. Soc. Transl. [1998], 186, 1-25
}

\begin{abstract}
There is a well developed and useful theory of Hamiltonian reduction for semidirect products, which applies to examples such as the heavy top, compressible fluids and MHD, which are governed by Lie-Poisson type equations. In this paper we study the Lagrangian analogue of this process and link it with the general theory of Lagrangian reduction; that is the reduction of variational principles. These reduced variational principles are interesting in their own right since they involve constraints on the allowed variations, analogous to
\end{abstract}

\footnotetext{
${ }^{*}$ Research partially supported by NSF grant DMS 96-33161 and DOE contract DE-FG0395ER25251

${ }^{\dagger}$ Research partially supported by NSF Grant DMS-9503273 and DOE contract DE-FG0395ER25245-A000.
} 
what one finds in the theory of nonholonomic systems with the Lagrange d'Alembert principle. In addition, the abstract theorems about circulation, what we call the Kelvin-Noether theorem, are given.

\section{Contents}

1 Introduction $\quad 2$

$\begin{array}{llr}2 & \text { Semidirect Product Reduction } & 7\end{array}$

3 Lagrangian Semidirect Product Theory 11

4 The Kelvin-Noether Theorem $\quad 14$

5 General Lagrangian Reduction. $\quad 17$

6 The Euler-Poincaré Equations via Lagrangian Reduction 22

\section{Introduction}

The main purpose of this paper is to develop the variational structure of the general Euler-Poincaré equations that are the Lagrangian counterpart to the Lie-Poisson equations associated with a semidirect product and to show how this is related to the general theory of Lagrangian reduction. We also want to explain the abstract notion of circulation that gives rise to a general Kelvin-Noether theorem.

To accomplish these goals, it will be convenient to first recall some simpler situations, namely the two general ways of abstracting the classical Euler equations for a fluid or a rigid body, namely Lie-Poisson systems on the dual of a Lie algebra and their Lagrangian counterpart, the "pure" Euler-Poincaré equations on a Lie algebra.

The Lie-Poisson Equations. The Lie-Poisson equations are Hamiltonian equations on the dual of a Lie algebra and represent an abstraction of the Euler equations for a rigid body (in body momentum representation) as well as the Euler equations for an ideal incompressible fluid (in spatial representation). This set up and its counterpart, the Euler-Poincaré equations on the Lie algebra are the basic ingredients used in the fundamental paper of Arnold [1966a].

Ignoring, for simplicity, function space technicalities in the infinite dimensional case (see Ebin and Marsden [1970] for one approach to dealing with them), we let $G$ be a Lie group with Lie algebra $\mathfrak{g}$ and let $F, K$ be real valued functions on the dual space $\mathfrak{g}^{*}$. Denoting elements of $\mathfrak{g}^{*}$ by $\mu$, let the functional derivative of $F$ at $\mu$ be the unique element $\delta F / \delta \mu$ of $\mathfrak{g}$ defined by

$$
\lim _{\varepsilon \rightarrow 0} \frac{1}{\varepsilon}[F(\mu+\varepsilon \delta \mu)-F(\mu)]=\left\langle\delta \mu, \frac{\delta F}{\delta \mu}\right\rangle,
$$


for all $\delta \mu \in \mathfrak{g}^{*}$, where $\langle$,$\rangle denotes the pairing between \mathfrak{g}^{*}$ and $\mathfrak{g}$. Define the $( \pm)$ Lie-Poisson brackets by

$$
\{F, G\}_{ \pm}(\mu)= \pm\left\langle\mu,\left[\frac{\delta F}{\delta \mu}, \frac{\delta G}{\delta \mu}\right]\right\rangle .
$$

These brackets, discovered by Lie [1890], make $\mathfrak{g}^{*}$ into a Poisson manifold and the Hamiltonian equations associated with a given Hamiltonian $H$ are called the Lie-Poisson equations. Thus, the Lie-Poisson equations are determined by $\dot{F}=$ $\{F, H\}$ for all $F$.

We recall these equations in the finite dimensional case in coordinates. Choose a basis $e_{1}, \ldots, e_{r}$ of $\mathfrak{g}$ (so $\left.\operatorname{dim} \mathfrak{g}=r\right)$. Define, as usual, the structure constants $C_{a b}^{d}$ of the Lie algebra by $\left[e_{a}, e_{b}\right]=C_{a b}^{d} e_{d}$, where $a, b$ run from 1 to $r$ and a sum on $d$ is understood. If $\xi \in \mathfrak{g}$, its components relative to this basis are denoted $\xi^{a}$, so $\xi=\xi^{a} e_{a}$. If $e^{1}, \ldots, e^{n}$ is the corresponding dual basis, and we write $\mu=\mu_{a} e^{a}$ (with a sum understood), the $( \pm)$ Lie-Poisson brackets become

$$
\{F, K\}_{ \pm}(\mu)= \pm C_{a b}^{d} \mu_{d} \frac{\partial F}{\partial \mu_{a}} \frac{\partial K}{\partial \mu_{b}} .
$$

The Lie-Poisson equations are

$$
\dot{\mu}_{a}=\left\{\mu_{a}, H\right\}_{ \pm}(\mu)= \pm C_{a b}^{d} \mu_{d} \frac{\partial H}{\partial \mu_{b}},
$$

or intrinsically,

$$
\dot{\mu}=\mp \operatorname{ad}_{\delta H / \delta \mu}^{*} \mu \text {. }
$$

Here $\operatorname{ad}_{\xi}: \mathfrak{g} \rightarrow \mathfrak{g}$ is the adjoint map $\eta \mapsto[\xi, \eta]$, and $\operatorname{ad}_{\xi}^{*}: \mathfrak{g}^{*} \rightarrow \mathfrak{g}^{*}$ is its dual.

However, the point of view of general Poisson manifolds for these systems is sometimes misleading, at least for applications in mechanics. What is more insightful, and which has its roots in Arnold [1966a] is the point of view that the Lie-Poisson brackets arise from canonical brackets on the cotangent bundle $T^{*} G$ by reduction. Namely, if we identify $\mathfrak{g}^{*}$ with the natural Poisson quotient $T^{*} G / G$ using the left action of $G$, we get the minus Lie-Poisson bracket, while the quotient with the right action gives the plus Lie-Poisson bracket.

The rigid body is naturally a left invariant system on $T^{*} \mathrm{SO}(3)$, while ideal fluids naturally give a right invariant system on the group of volume preserving diffeomorphisms of the fluid within the container.

Poincaré and the Euler equations. Poincaré's work on the gravitating fluid problem continues the line of investigation begun by MacLaurin, Jacobi and Riemann and was a natural precursor to his famous paper, Poincaré [1901], in which he laid out the basic equations of Euler type on Lie algebras. He was certainly aware that this formalism includes the rigid body, heavy top and ideal fluids as special cases.

To state the Euler-Poincaré equations, let $\mathfrak{g}$ be a given Lie algebra and let $l$ : $\mathfrak{g} \rightarrow \mathbb{R}$ be a given function (the Lagrangian), let $\xi$ be a point in $\mathfrak{g}$ and let $f \in \mathfrak{g}^{*}$ be a 
given force (the nature of which we shall explicate later). Then the Euler-Poincaré equations are the following equations for the evolution of the basic variable $\xi$ :

$$
\frac{d}{d t} \frac{\delta l}{\delta \xi}=\operatorname{ad}_{\xi}^{*} \frac{\delta l}{\delta \xi}+f
$$

The notation is as follows: $\delta l / \delta \xi \in \mathfrak{g}^{*}$ is the derivative of $l$ with respect to $\xi$ - the dual to the derivative notation used for the Lie-Poisson equations.

As with the Lie-Poisson setting, these equations are valid for either finite or infinite dimensional Lie algebras. For fluids, Poincaré was aware that one needs to use infinite dimensional Lie algebras, as is clear from Poincaré [1910]. The equations also come in two flavors, one for left invariant systems (the one given) and one for right invariant systems in which case one changes the sign in front of the adjoint operator (or changes conventions in the Lie algebra brackets).

In the finite dimensional case, the equations read

$$
\frac{d}{d t} \frac{\partial l}{\partial \xi^{d}}=C_{a d}^{b} \frac{\partial l}{\partial \xi^{b}} \xi^{a}+f_{d}
$$

For example, consider the Lie algebra $\mathbb{R}^{3}$ with the usual vector cross product. (Of course, this is the Lie algebra of the proper rotation group in $\mathbb{R}^{3}$.) For $l: \mathbb{R}^{3} \rightarrow \mathbb{R}$, the Euler-Poincaré equations become

$$
\frac{d}{d t} \frac{\partial l}{\partial \Omega}=\frac{\partial l}{\partial \Omega} \times \Omega+f
$$

which generalize the Euler equations for rigid body motion to include external forces and Lagrangians that needn't be quadratic.

These equations were written down for a certain class of Lagrangians $l$ by Lagrange [1788, Volume 2, Equation A on p. 212], while it was Poincaré [1901] who generalized them (without reference to the ungeometric Lagrange!) to any Lie algebra. However, Lagrange did grapple (in much of volume 2 of his treatise) with the derivation and deeper understanding of the nature of these equations. While Poincaré may have understood how to derive them from other principles, he did not reveal this.

Of course, there was a lot of mechanics going on in the decades leading up to Poincaré's time. It is a curious historical fact that the Euler-Poincaré equations were not pursued extensively and systematically until rather recently. While many authors mentioned them (see, e.g., Hamel [1904, 1949] and Chetayev [1941]), it was not until the Arnold school that a deeper understanding was achieved and was used for purposes of hydrodynamical stability (see Arnold [1966b, 1988] and Arnold and Khesin [1992, 1997]).

We now recall the derivation of the "pure" Euler-Poincaré equations (i.e., the Euler-Poincaré equations with no forcing or advected terms) for left-invariant Lagrangians on Lie groups (see Marsden and Scheurle [1993a,b], Marsden and Ratiu [1994], and Bloch et al. [1996]). The Lagrangian counterpart to the Lie-Poisson reduction of Poisson manifolds mentioned above is the following: 
Theorem 1.1 Let $G$ be a Lie group and $L: T G \rightarrow \mathbb{R}$ a left (right) invariant Lagrangian. Let $l: \mathfrak{g} \rightarrow \mathbb{R}$ be its restriction to the tangent space at the identity. For a curve $g(t) \in G$, let $\xi(t)=g(t)^{-1} \dot{g}(t)$ (respectively $\left.\xi(t)=\dot{g}(t) g(t)^{-1}\right)$. Then the following are equivalent:

i Hamilton's principle

$$
\delta \int_{a}^{b} L(g(t), \dot{g}(t)) d t=0
$$

holds, as usual, for variations $\delta g(t)$ of $g(t)$ vanishing at the endpoints.

ii The curve $g(t)$ satisfies the Euler-Lagrange equations for $L$ on $G$.

iii The "variational" principle

$$
\delta \int_{a}^{b} l(\xi(t)) d t=0
$$

holds on $\mathfrak{g}$, using variations of the form

$$
\delta \xi=\dot{\eta} \pm[\xi, \eta]
$$

where $\eta$ vanishes at the endpoints (+ corresponds to left invariance and - to right invariance). ${ }^{1}$

iv The pure Euler-Poincaré equations hold

$$
\frac{d}{d t} \frac{\delta l}{\delta \xi}= \pm \operatorname{ad}_{\xi}^{*} \frac{\delta l}{\delta \xi}
$$

We make some comments on the proof. First of all, the equivalence of $\mathbf{i}$ and ii holds on the tangent bundle of any configuration manifold $Q$, by the general Hamilton principle. To see that ii and iv are equivalent, one needs to compute the variations $\delta \xi$ induced on $\xi=g^{-1} \dot{g}=T L_{g^{-1}} \dot{g}$ by a variation of $g$. We will do this for matrix groups; see Bloch, Krishnaprasad, Marsden, and Ratiu [1994, 1996] for the general case. To calculate this, we need to differentiate $g^{-1} \dot{g}$ in the direction of a variation $\delta g$. If $\delta g=d g / d \epsilon$ at $\epsilon=0$, where $g$ is extended to a curve $g_{\epsilon}$, then,

$$
\delta \xi=\frac{d}{d \epsilon} g^{-1} \frac{d}{d t} g,
$$

while if $\eta=g^{-1} \delta g$, then

$$
\dot{\eta}=\frac{d}{d t} g^{-1} \frac{d}{d \epsilon} g .
$$

The difference $\delta \xi-\dot{\eta}$ is thus the commutator $[\xi, \eta]$.

\footnotetext{
${ }^{1}$ Because there are constraints on the variations, this principle is more like a Lagrange d'Alembert principle, which is why we put "variational" in quotes. Of course such problems are not literally variational.
} 
To complete the proof, we show the equivalence of iii and iv. Indeed, using the definitions and integrating by parts,

$$
\begin{aligned}
\delta \int l(\xi) d t & =\int \frac{\delta l}{\delta \xi} \delta \xi d t=\int \frac{\delta l}{\delta \xi}\left(\dot{\eta}+\operatorname{ad}_{\xi} \eta\right) d t \\
& =\int\left[-\frac{d}{d t}\left(\frac{\delta l}{\delta \xi}\right)+\operatorname{ad}_{\xi}^{*} \frac{\delta l}{\delta \xi}\right] \eta d t
\end{aligned}
$$

so the result follows.

Since the Euler-Lagrange and Hamilton equations on $T Q$ and $T^{*} Q$ are equivalent for regular Lagrangians or Hamiltonians, it follows that the Lie-Poisson and EulerPoincaré equations are also equivalent under a regularity condition. To see this directly, we make the following Legendre transformation from $\mathfrak{g}$ to $\mathfrak{g}^{*}$ :

$$
\mu=\frac{\delta l}{\delta \xi}, \quad h(\mu)=\langle\mu, \xi\rangle-l(\xi)
$$

Note that

$$
\frac{\delta h}{\delta \mu}=\xi+\left\langle\mu, \frac{\delta \xi}{\delta \mu}\right\rangle-\left\langle\frac{\delta l}{\delta \xi}, \frac{\delta \xi}{\delta \mu}\right\rangle=\xi
$$

and so it is now clear that the Lie-Poisson and Euler-Poincaré equations are equivalent. However, we shall see shortly that this does not work for examples like the heavy top, MHD etc., when we write the equations as Lie-Poisson equations on the dual of a semidirect product; the reason is that in these examples, the Hamiltonian is degenerate, i.e., if the matrix of second derivatives $H_{p_{i} p_{j}}$ is singular. This is a fundamental obstacle and may be viewed as one reason that the Lagrangian version of the semidirect product theory is interesting. We shall come to this shortly.

We close this section by mentioning that many other systems can be put into Euler-Poincaré form. For example, this can be done for the KdV equations as has been shown by Ovsienko and Khesin [1987] (an account may be found in Marsden and Ratiu [1994]). The same has been shown for many other shallow water equations and equations of continuum mechanics, including the equations of plasma physics (cf. Holm, Marsden and Ratiu [1997] and Cendra, Holm, Hoyle and Marsden [1997]).

Plan of the paper. With the above background in hand, we first set the stage for the main goal of the paper, the comparison of the general Euler-Poincaré equations with Lagrangian reduction, by describing some of the ingredients in more detail. The first of these is the Hamiltonian theory of semidirect product reduction in $\S 2$. The next main ingredient, in $\S 3$, is the derivation of the general Euler-Poincaré equations by the method of reduction of variational principles. In $\S 4$ we give the Kelvin-Noether theorem; while not essential to the main goal of the paper, it is a fundamental result for the Euler-Poincaré equations, so it is given for completeness. In $\S 5$ we describe the general theory of Lagrangian reduction and illustrate the methods with Wong's equations and use Lagrangian reduction to give a simple proof of the falling cat theorem of Montgomery. Finally, in $\S 6$, we show how the Euler-Poincaré equations are linked with the general theory of Lagrangian reduction. 


\section{Semidirect Product Reduction}

Before getting to the Lagrangian analogue of semidirect product reduction, it is useful to summarize the Hamiltonian theory.

The theory begins with the understanding of various examples, such as the heavy top, ideal compressible fluids and MHD (magnetohydrodynamics). Building on these examples, the general study of Lie-Poisson equations for systems on the dual of a semidirect product Lie algebra was developed by many authors such as Sudarshan and Mukunda [1974], Vinogradov and Kupershmidt [1977], Ratiu [1980], Guillemin and Sternberg [1980], Ratiu [1981, 1982], Marsden [1982], Marsden, Weinstein, Ratiu, Schmidt and Spencer [1983], Holm and Kupershmidt [1983], Kupershmidt and Ratiu [1983], Holmes and Marsden [1983], Marsden, Ratiu and Weinstein [1984a,b], Guillemin and Sternberg [1980, 1984], Holm, Marsden, Ratiu and Weinstein [1985], Abarbanel, Holm, Marsden, and Ratiu [1986], Leonard and Marsden [1997] and Marsden, Misiolek, Perlmutter and Ratiu [1997]. As these and related references show, these equations apply to a wide variety of systems such as the heavy top, compressible flow, stratified incompressible flow, MHD and the dynamics of underwater vehicles.

Generalities on Semidirect Products. We first recall some definitions. Let $V$ be a vector space and assume that the Lie group $G$ acts on the left by linear maps on $V$ (and hence $G$ also acts on on the left on its dual space $V^{*}$ ). As sets, the semidirect product $S=G(S)$ is the Cartesian product $S=G \times V$ whose group multiplication is given by

$$
\left(g_{1}, v_{1}\right)\left(g_{2}, v_{2}\right)=\left(g_{1} g_{2}, v_{1}+g_{1} v_{2}\right),
$$

where the action of $g \in G$ on $v \in V$ is denoted simply as $g v$. The identity element is $(e, 0)$ where $e$ is the identity in $G$ and inversion is $(g, v)^{-1}=\left(g^{-1},-g^{-1} v\right)$.

The Lie algebra of $S$ is the semidirect product Lie algebra, $\mathfrak{s}=\mathfrak{g} S V$, with the bracket

$$
\left[\left(\xi_{1}, v_{1}\right),\left(\xi_{2}, v_{2}\right)\right]=\left(\left[\xi_{1}, \xi_{2}\right], \xi_{1} v_{2}-\xi_{2} v_{1}\right),
$$

where we denote actions, such as the induced action of $\mathfrak{g}$ on $V$ by concatenation, as in $\xi_{1} v_{2}$.

The adjoint and the coadjoint actions for semidirect products are given by (see, e.g., Marsden, Ratiu and Weinstein [1984a,b]):

$$
(g, v)(\xi, u)=(g \xi, g u-(g \xi) v)
$$

and

$$
(g, v)(\mu, a)=\left(g \mu+\rho_{v}^{*}(g a), g a\right),
$$

where $(g, v) \in S=G \times V,(\xi, u) \in \mathfrak{s}=\mathfrak{g} \times V,(\mu, a) \in \mathfrak{s}^{*}=\mathfrak{g}^{*} \times V^{*}, g \xi=\operatorname{Ad}_{g} \xi$, $g \mu=\mathrm{Ad}_{g^{-1}}^{*} \mu, g a$ denotes the induced left action of $g$ on $a$ (the left action of $G$ on $V$ induces a left action of $G$ on $V^{*}$ — the inverse of the transpose of the action on 
$V), \rho_{v}: \mathfrak{g} \rightarrow V$ is the linear map given by $\rho_{v}(\xi)=\xi v$, and $\rho_{v}^{*}: V^{*} \rightarrow \mathfrak{g}^{*}$ is its dual.

For $a \in V^{*}$, we shall write, for notational convenience,

$$
\rho_{v}^{*} a=v \diamond a \in \mathfrak{g}^{*},
$$

which is a bilinear operation in $v$ and $a$. Continuing to employ the concatenation notation for Lie group or algebra actions, the identity

$$
\langle\eta a, v\rangle=-\langle v \diamond a, \eta\rangle
$$

for all $v \in V, a \in V^{*}$ and $\eta \in \mathfrak{g}$ is another way to write the definition of $v \diamond a \in \mathfrak{g}^{*}$. Using this notation, the coadjoint action reads $(g, v)(\mu, a)=(g \mu+v \diamond(g a), g a)$.

When working with various models of continuum mechanics and plasmas it is convenient to work with right representations of $G$ on the vector space $V$ (as in, for example, Holm, Marsden and Ratiu [1986]). In this context of course the above formalism must be suitably modified.

Lie-Poisson Brackets and Hamiltonian Vector Fields. For a left representation of $G$ on $V$ the \pm Lie-Poisson bracket of two functions $f, k: \mathfrak{s}^{*} \rightarrow \mathbb{R}$ is given by

$$
\{f, k\}_{ \pm}(\mu, a)= \pm\left\langle\mu,\left[\frac{\delta f}{\delta \mu}, \frac{\delta k}{\delta \mu}\right]\right\rangle \pm\left\langle a, \frac{\delta f}{\delta \mu} \frac{\delta k}{\delta a}-\frac{\delta k}{\delta \mu} \frac{\delta f}{\delta a}\right\rangle
$$

where $\delta f / \delta \mu \in \mathfrak{g}$, and $\delta f / \delta a \in V$ are the functional derivatives of $f$. The Hamiltonian vector field of $h: \mathfrak{s}^{*} \rightarrow \mathbb{R}$ has the expression

$$
X_{h}(\mu, a)=\mp\left(\operatorname{ad}_{\delta h / \delta \mu}^{*} \mu-\frac{\delta h}{\delta a} \diamond a,-\frac{\delta h}{\delta \mu} a\right)
$$

Thus, Hamilton's equations (the Lie-Poisson equations) on the dual of a semidirect product are given by

$$
\begin{aligned}
\dot{\mu} & =\mp \operatorname{ad}_{\delta h / \delta \mu}^{*} \mu \pm \frac{\delta h}{\delta a} \diamond a, \\
\dot{a} & = \pm \frac{\delta h}{\delta \mu} a,
\end{aligned}
$$

where overdot denotes time derivative. Again, for right representations of $G$ on $V$ the above formulae must be appropriately modified.

Symplectic Actions by Semidirect Products. We consider a (left) symplectic action of $S$ on a symplectic manifold $P$ and assume that this action has an equivariant momentum map $\mathbf{J}_{S}: P \rightarrow \mathfrak{s}^{*}$. Since $V$ is a (normal) subgroup of $S$, it also acts on $P$ and has a momentum map $\mathbf{J}_{V}: P \rightarrow V^{*}$ given by $\mathbf{J}_{V}=i_{V}^{*} \circ \mathbf{J}_{S}$, where $i_{V}: V \rightarrow \mathfrak{s}$ is the inclusion $v \mapsto(0, v)$ and $i_{V}^{*}: \mathfrak{s}^{*} \rightarrow V^{*}$ is its dual. We think of this merely as saying that $\mathbf{J}_{V}$ is the second component of $\mathbf{J}_{S}$. 
We can regard $G$ as a subgroup of $S$ by $g \mapsto(g, 0)$. Thus, $G$ also has a momentum map that is the first component of $\mathbf{J}_{S}$ but this will play a secondary role in what follows. On the other hand, equivariance of $\mathbf{J}_{S}$ under $G$ implies the following relation for $\mathbf{J}_{V}$ :

$$
\mathbf{J}_{V}(g z)=g \mathbf{J}_{V}(z)
$$

where we denote the appropriate action of $g \in G$ on an element by concatenation, as before. To prove (2.10), one uses the fact that for the coadjoint action of $S$ on $\mathfrak{s}^{*}$ the second component is just the dual of the given action of $G$ on $V$.

The Classical Semidirect Product Reduction Theorem. In a number of interesting applications such as compressible fluids, the heavy top, MHD, etc., one has two symmetry groups that do not commute and commuting reduction by stages (a theorem from Marsden and Weinstein [1974]) does not apply. In this more general situation, it matters in what order one performs the reduction, which occurs, in particular for semidirect products. The main result covering the case of semidirect products has a complicated history, some of which has been sketched; we follow the version of Marsden, Ratiu and Weinstein [1984a,b].

The semidirect product reduction theorem states, roughly speaking, that for the semidirect product $S=G$ S $V$ where $G$ is a group acting on a vector space $V$ and $S$ is the semidirect product, one can first reduce $T^{*} S$ by $V$ and then by $G$ and obtain the same result as reducing by $S$. As above, we let $\mathfrak{s}=\mathfrak{g}(S)$ denote the Lie algebra of $S$. The precise statement is as follows.

Theorem 2.1 (Semidirect Product Reduction Theorem.) Let $S=G(S)$, choose $\sigma=(\mu, a) \in \mathfrak{g}^{*} \times V^{*}$, and reduce $T^{*} S$ by the action of $S$ at $\sigma$ giving the coadjoint orbit $\mathcal{O}_{\sigma}$ through $\sigma \in \mathfrak{s}^{*}$. There is a symplectic diffeomorphism between $\mathcal{O}_{\sigma}$ and the reduced space obtained by reducing $T^{*} G$ by the subgroup $G_{a}$ (the isotropy of $G$ for its action on $V^{*}$ at the point $a \in V^{*}$ ) at the point $\mu_{a} \in \mathfrak{g}_{a}^{*}$ defined by restriction: $\mu_{a}=\mu \mid \mathfrak{g}_{a}$, where $\mathfrak{g}_{a}$ is the Lie algebra of $G_{a}$.

Reduction by Stages. The preceding result is a special case of a general theorem on reduction by stages for semidirect products acting on a symplectic manifold (See Marsden, Misiolek, Perlmutter and Ratiu [1997] for this and more general results dealing with group extensions and see Leonard and Marsden [1997] for an application to underwater vehicle dynamics.)

As above, consider a symplectic action of $S$ on a symplectic manifold $P$ and assume that this action has an equivariant momentum map $\mathbf{J}_{S}: P \rightarrow \mathfrak{s}^{*}$. As we have explained, the momentum map for the action of $V$ is the map $\mathbf{J}_{V}: P \rightarrow V^{*}$ given by $\mathbf{J}_{V}=i_{V}^{*} \circ \mathbf{J}_{S}$

We carry out the symplectic reduction of $P$ by $S$ at a regular value $\sigma=(\mu, a)$ of the momentum map $\mathbf{J}_{S}$ for $S$ in two stages. First, reduce $P$ by $V$ at the value $a$ (assume it to be a regular value) to get the reduced space $P_{a}:=\mathbf{J}_{V}^{-1}(a) / V$. Second, form the group $G_{a}$ consisting of elements of $G$ that leave the point $a$ fixed using the action of $G$ on $V^{*}$. One shows (and this step is not trivial) that the group $G_{a}$ 
acts on $P_{a}$ and has an induced equivariant momentum map $\mathbf{J}_{a}: P_{a} \rightarrow \mathfrak{g}_{a}^{*}$, where $\mathfrak{g}_{a}$ is the Lie algebra of $G_{a}$, so one can reduce $P_{a}$ at the point $\mu_{a}:=\mu \mid \mathfrak{g}_{a}$ to get the reduced space $\left(P_{a}\right)_{\mu_{a}}=\mathbf{J}_{a}^{-1}\left(\mu_{a}\right) /\left(G_{a}\right)_{\mu_{a}}$.

Theorem 2.2 (Reduction by Stages for Semidirect Products.) The reduced space $\left(P_{a}\right)_{\mu_{a}}$ is symplectically diffeomorphic to the reduced space $P_{\sigma}$ obtained by reducing $P$ by $S$ at the point $\sigma=(\mu, a)$.

Semidirect Product Reduction of Dynamics. There is a technique for reducing dynamics that is associated with the geometry of the semidirect product reduction theorem. We start with a Hamiltonian $H_{a_{0}}$ on $T^{*} G$ that depends parametrically on a variable $a_{0} \in V^{*}$. The Hamiltonian, regarded as a map $H: T^{*} G \times V^{*} \rightarrow \mathbb{R}$ is assumed to be invariant on $T^{*} G$ under the action of $G$ on $T^{*} G \times V^{*}$. This condition is equivalent to the invariance of the function $H$ defined on $T^{*} S=T^{*} G \times V \times V^{*}$ extended to be constant in the variable $V$ under the action of the semidirect product. By the semidirect product reduction theorem, the dynamics of $H_{a_{0}}$ reduced by $G_{a_{0}}$, the isotropy group of $a_{0}$, is symplectically equivalent to Lie-Poisson dynamics on $\mathfrak{s}^{*}=\mathfrak{g}^{*} \times V^{*}$. This Lie-Poisson dynamics is given by the equations (2.8) and (2.9) for the function $h(\mu, a)=H\left(\alpha_{g}, g^{-1} a\right)$ where $\mu=g^{-1} \alpha_{g}$.

Cotangent bundle reduction. It will be useful to recall a few additional facts about cotangent bundle reduction. Following standard notation consistent with what we have already used, a symplectic reduced space at momentum value $\mu$ is denoted $P_{\mu}:=\mathbf{J}^{-1}(\mu) / G_{\mu}$. For cotangent bundle reduction we are considering $P=T^{*} Q$ and the action of a Lie group $G$ on $Q$ with the standard momentum map. The simplest case of cotangent bundle reduction is reduction at the momentum value zero in which case one has $\left(T^{*} Q\right)_{\mu=0}=T^{*}(Q / G)$, the latter with the canonical symplectic form. If $G$ is abelian, then $\left(T^{*} Q\right)_{\mu} \cong T^{*}(Q / G)$ but the latter has a symplectic structure modified by magnetic terms; that is, by the curvature of the mechanical connection. This abelian version of cotangent bundle reduction was developed by Smale [1970] and Satzer [1977] and was generalized to the nonabelian case in Abraham and Marsden [1978]. Kummer [1981] introduced the interpretations of these results in terms of the mechanical connection. This set up was effectively used, for example, in Guichardet [1984] and Iwai [1987] to study geometric phases in classical molecular dynamics. For additional information on the cotangent bundle reduction theorem, see Marsden [1992].

When combined with the cotangent bundle reduction theorem, the semidirect product reduction theorem is a useful tool. For example this shows directly that the generic coadjoint orbits for the Euclidean group are cotangent bundles of spheres with the associated coadjoint orbit symplectic structure given by the canonical structure plus a magnetic term. In fact, this technique allows one to understand the analogue of this geometrical structure for general semidirect products.

The "bundle picture" begun by these early works was significantly developed by Montgomery, Marsden and Ratiu [1984] and by Montgomery [1986], and was 
motivated by work of Weinstein and Sternberg on Wong's equations (the equations for a particle moving in a Yang-Mills field). We shall come to the Lagrangian counterpart of this theory shortly.

\section{Lagrangian Semidirect Product Theory}

Introduction. Despite all the activity on the Hamiltonian theory of semidirect products, little attention was paid to the corresponding Lagrangian side. Now that Lagrangian reduction is maturing (see Marsden and Scheurle [1993a,b]) and useful applications are emerging such as to Hamilton's principle asymptotics (see Holm [1996]) and to numerical algorithms (Wendlandt and Marsden [1997] and Marsden, Patrick and Shkoller [1997]), it is appropriate to consider the corresponding Lagrangian question more deeply.

The theory is entirely based on variational principles with symmetry. Consistent with the variational formulation, note that none of the theorems in this section require that the Lagrangian be nondegenerate. The theory is not dependent on the previous Hamiltonian formulation, although we shall, of course, make links with it under the appropriate regularity assumptions.

The theorems that follow are modeled after the reduction theorem for the pure Euler-Poincaré equations given in the introduction, although, as we shall explain, they are not literally special cases of it. As in the Hamiltonian case, the main distinction between the pure Euler-Poincaré equations and the general ones is the presence of advected quantities, such as the body representation of the direction of gravity in the heavy top and the density in compressible fluids.

Abstractly, these advected quantities are reflected by the fact that the Lagrangian $L$ and its reduction $l$, depend on another parameter $a \in V^{*}$, where, as in the Hamiltonian case, $V$ is a representation space for the Lie group $G$ and $L$ has an invariance property relative to both arguments.

As we shall see shortly, the resulting Euler-Poincaré equations are not the same as the pure Euler-Poincaré equations for the semidirect product Lie algebra $\mathfrak{g}\left(\mathrm{S} V^{*}\right.$.

The basic ingredients. We begin with a left representation of Lie group $G$ on the vector space $V$ and $G$ acts in the natural way on the left on $T G \times V^{*}: h\left(v_{g}, a\right)=$ $\left(h v_{g}, h a\right)$. We assume that we have a $L: T G \times V^{*} \rightarrow \mathbb{R}$ is left $G$-invariant. In particular, if $a_{0} \in V^{*}$, define the Lagrangian $L_{a_{0}}: T G \rightarrow \mathbb{R}$ by $L_{a_{0}}\left(v_{g}\right)=L\left(v_{g}, a_{0}\right)$. Then $L_{a_{0}}$ is left invariant under the lift to $T G$ of the left action of $G_{a_{0}}$ on $G$, where $G_{a_{0}}$ is the isotropy group of $a_{0}$.

Left $G$-invariance of $L$ permits us to define the reduced Lagrangian $l: \mathfrak{g} \times V^{*} \rightarrow \mathbb{R}$ by $l\left(g^{-1} v_{g}, g^{-1} a\right)=L\left(v_{g}, a\right)$. Conversely, this relation defines for any $l: \mathfrak{g} \times V^{*} \rightarrow \mathbb{R}$ a left $G$-invariant function $L: T G \times V^{*} \rightarrow \mathbb{R}$.

For a curve $g(t) \in G$, let $\xi(t):=g(t)^{-1} \dot{g}(t)$ and define the curve $a(t)$ as the unique solution of the following linear differential equation with time dependent coefficients $\dot{a}(t)=-\xi(t) a(t)$, with initial condition $a(0)=a_{0}$. The solution can be written as $a(t)=g(t)^{-1} a_{0}$. 
The Euler-Poincaré equations. The generalization of the pure Euler-Poincaré theorem is the following.

Theorem 3.1 With the preceding notation, the following are equivalent:

i With $a_{0}$ held fixed, Hamilton's variational principle

$$
\delta \int_{t_{1}}^{t_{2}} L_{a_{0}}(g(t), \dot{g}(t)) d t=0
$$

holds, for variations $\delta g(t)$ of $g(t)$ vanishing at the endpoints.

ii $g(t)$ satisfies the Euler-Lagrange equations for $L_{a_{0}}$ on $G$.

iii The constrained variational principle (of Lagrange d'Alembert type)

$$
\delta \int_{t_{1}}^{t_{2}} l(\xi(t), a(t)) d t=0
$$

holds on $\mathfrak{g} \times V^{*}$, using variations of $\xi$ and a of the form

$$
\delta \xi=\dot{\eta}+[\xi, \eta], \quad \delta a=-\eta a,
$$

where $\eta(t) \in \mathfrak{g}$ vanishes at the endpoints.

iv The Euler-Poincaré equations hold on $\mathfrak{g} \times V^{*}$

$$
\frac{d}{d t} \frac{\delta l}{\delta \xi}=\operatorname{ad}_{\xi}^{*} \frac{\delta l}{\delta \xi}+\frac{\delta l}{\delta a} \diamond a, \quad \text { where } \quad \dot{a}(t)=-\xi(t) a(t) .
$$

Proof. The equivalence of $\mathbf{i}$ and $\mathbf{i i}$ holds for any configuration manifold and so, in particular, it holds in this case.

Next we show the equivalence of iii and iv. Indeed, using the definitions, integrating by parts, and taking into account that $\eta\left(t_{1}\right)=\eta\left(t_{2}\right)=0$, we compute the variation of the integral to be

$$
\begin{aligned}
\delta \int_{t_{1}}^{t_{2}} l(\xi(t), a(t)) d t & =\int_{t_{1}}^{t_{2}}\left(\left\langle\frac{\delta l}{\delta \xi}, \delta \xi\right\rangle+\left\langle\delta a, \frac{\delta l}{\delta a}\right\rangle\right) d t \\
& =\int_{t_{1}}^{t_{2}}\left(\left\langle\frac{\delta l}{\delta \xi}, \dot{\eta}+\operatorname{ad}_{\xi} \eta\right\rangle-\left\langle\eta a, \frac{\delta l}{\delta a}\right\rangle\right) d t \\
& =\int_{t_{1}}^{t_{2}}\left(\left\langle-\frac{d}{d t}\left(\frac{\delta l}{\delta \xi}\right)+\operatorname{ad}_{\xi}^{*} \frac{\delta l}{\delta \xi}, \eta\right\rangle+\left\langle\frac{\delta l}{\delta a} \diamond a, \eta\right\rangle\right) d t \\
& =\int_{t_{1}}^{t_{2}}\left\langle-\frac{d}{d t}\left(\frac{\delta l}{\delta \xi}\right)+\operatorname{ad}_{\xi}^{*} \frac{\delta l}{\delta \xi}+\frac{\delta l}{\delta a} \diamond a, \eta\right\rangle d t
\end{aligned}
$$

and so the result follows.

Finally we show that $\mathbf{i}$ and iii are equivalent. First note that the $G$-invariance of $L: T G \times V^{*} \rightarrow \mathbb{R}$ and the definition of $a(t)=g(t)^{-1} a_{0}$ imply that the integrands 
in (3.1) and (3.2) are equal. However, all variations $\delta g(t) \in T G$ of $g(t)$ with fixed endpoints induce and are induced by variations $\delta \xi(t) \in \mathfrak{g}$ of $\xi(t)$ of the form $\delta \xi=$ $\dot{\eta}+[\xi, \eta]$ with $\eta(t) \in \mathfrak{g}$ vanishing at the endpoints; the relation between $\delta g(t)$ and $\eta(t)$ is given by $\eta(t)=g(t)^{-1} \delta g(t)$. This is the content of the following lemma (which is elementary for matrix groups) proved in Bloch et al. [1996].

Lemma 3.2 Let $g: U \subset \mathbb{R}^{2} \rightarrow G$ be a smooth map and denote its partial derivatives by $\xi(t, \varepsilon)=T L_{g(t, \varepsilon)^{-1}}(\partial g(t, \varepsilon) / \partial t)$ and $\eta(t, \varepsilon)=T L_{g(t, \varepsilon)^{-1}}(\partial g(t, \varepsilon) / \partial \varepsilon)$. Then

$$
\frac{\partial \xi}{\partial \varepsilon}-\frac{\partial \eta}{\partial t}=[\xi, \eta]
$$

Conversely, if $U$ is simply connected and $\xi, \eta: U \rightarrow \mathfrak{g}$ are smooth functions satisfying (3.5) then there exists a smooth function $g: U \rightarrow G$ such that $\xi(t, \varepsilon)=$ $T L_{g(t, \varepsilon)^{-1}}(\partial g(t, \varepsilon) / \partial t)$ and $\eta(t, \varepsilon)=T L_{g(t, \varepsilon)^{-1}}(\partial g(t, \varepsilon) / \partial \varepsilon)$.

Thus, if $\mathbf{i}$ holds, we define $\eta(t)=g(t)^{-1} \delta g(t)$ for a variation $\delta g(t)$ with fixed endpoints. Then if we let $\delta \xi=g(t)^{-1} \dot{g}(t)$, we have by the above proposition $\delta \xi=\dot{\eta}+$ $[\xi, \eta]$. In addition, the variation of $a(t)=g(t)^{-1} a_{0}$ is $\delta a(t)=-\eta(t) a(t)$. Conversely, if $\delta \xi=\dot{\eta}+[\xi, \eta]$ with $\eta(t)$ vanishing at the endpoints, we define $\delta g(t)=g(t) \eta(t)$ and the above proposition guarantees then that this $\delta g(t)$ is the general variation of $g(t)$ vanishing at the endpoints. From $\delta a(t)=-\eta(t) a(t)$ it follows that the variation of $g(t) a(t)=a_{0}$ vanishes, which is consistent with the dependence of $L_{a_{0}}$ only on $g(t), \dot{g}(t)$

Remark. The Euler-Poincaré equations are not the pure Euler-Poincaré equations because we are not regarding $\mathfrak{g} \times V^{*}$ as a Lie algebra. Rather these equations are thought of as a generalization of the classical Euler-Poisson equations for a heavy top, but written in body angular velocity variables. Some authors may thus prefer the term Euler-Poisson-Poincaré equations for these equations. The following argument shows that these Euler-Poincaré equations (3.4) are not the pure EulerPoincaré equations for the semidirect product Lie algebra $\mathfrak{g} \subseteq V^{*}$. Indeed, by (1.9) the pure Euler-Poincaré equations

$$
\frac{d}{d t} \frac{\delta l}{\delta(\xi, a)}=\operatorname{ad}_{(\xi, a)}^{*} \frac{\delta l}{\delta(\xi, a)}, \quad(\xi, a) \in \mathfrak{g}\left(V^{*}\right.
$$

for $l: \mathfrak{g}(\mathrm{S}) V^{*} \rightarrow \mathbb{R}$ become

$$
\frac{d}{d t} \frac{\delta l}{\delta \xi}=\operatorname{ad}_{\xi}^{*} \frac{\delta l}{\delta \xi}+\frac{\delta l}{\delta a} \diamond a, \quad \frac{d}{d t} \frac{\delta l}{\delta a}=-\xi \frac{\delta l}{\delta a},
$$

which is a different system from that given by the Euler-Poincaré equation (3.4) and $\dot{a}=-\xi a$, even though the first equations of both systems are identical. 
The Legendre Transformation. As we explained earlier, one normally thinks of passing from Euler-Poincaré equations on a Lie algebra $\mathfrak{g}$ to Lie-Poisson equations on the dual $\mathfrak{g}^{*}$ by means of the Legendre transformation. In our case, we start with a Lagrangian on $\mathfrak{g} \times V^{*}$ and perform a partial Legendre transformation in the variable $\xi$ only, by writing

$$
\mu=\frac{\delta l}{\delta \xi}, \quad h(\mu, a)=\langle\mu, \xi\rangle-l(\xi, a) .
$$

A simple calculation shows that $\delta h / \delta \mu=\xi$, and $\delta h / \delta a=-\delta l / \delta a$, so that (3.4) and $\dot{a}(t)=-\xi(t) a(t)$ imply (2.7) for the minus Lie-Poisson bracket (that is, the sign + in (2.7)). If this Legendre transformation is invertible, then we can also pass from the minus Lie-Poisson equations (2.7) to the Euler-Poincaré equations (3.4) together with the equations $\dot{a}(t)=-\xi(t) a(t)$.

\section{The Kelvin-Noether Theorem}

In this section, we derive a version of the Noether theorem that holds for solutions of the Euler-Poincaré equations. Our formulation is motivated and designed for continuum theories (and hence the name Kelvin-Noether), but it holds in a more general situation. Of course it is well known (going back at least to the work of Arnold [1966a]) that the Kelvin circulation theorem for ideal flow is closely related to the Noether theorem applied to continua using the particle relabeling symmetry group.

The Kelvin-Noether Quantity. Start with a Lagrangian $L_{a_{0}}$ depending on a parameter $a_{0} \in V^{*}$ as above. Consider a manifold $\mathcal{C}$ on which $G$ acts (we assume this is also a left action) and suppose we have an equivariant map $\mathcal{K}: \mathcal{C} \times V^{*} \rightarrow \mathfrak{g}^{* *}$.

In the case of continuum theories, the space $\mathcal{C}$ may be chosen to be a loop space and $\langle\mathcal{K}(c, a), \mu\rangle$ for $c \in \mathcal{C}$ and $\mu \in \mathfrak{g}^{*}$ will be a circulation. This class of examples also shows why we do not want to identify the double dual $\mathfrak{g}^{* *}$ with $\mathfrak{g}$.

Define the Kelvin-Noether quantity $I: \mathcal{C} \times \mathfrak{g} \times V^{*} \rightarrow \mathbb{R}$ by

$$
I(c, \xi, a)=\left\langle\mathcal{K}(c, a), \frac{\delta l}{\delta \xi}(\xi, a)\right\rangle .
$$

Theorem 4.1 (Kelvin-Noether) Fixing $c_{0} \in \mathcal{C}$, let $\xi(t), a(t)$ satisfy the EulerPoincaré equations and define $g(t)$ to be the solution of $\dot{g}(t)=g(t) \xi(t)$ and, say, $g(0)=e$. Let $c(t)=g(t)^{-1} c_{0}$ and $I(t)=I(c(t), \xi(t), a(t))$. Then

$$
\frac{d}{d t} I(t)=\left\langle\mathcal{K}(c(t), a(t)), \frac{\delta l}{\delta a} \diamond a\right\rangle .
$$


Proof. First of all, write $a(t)=g(t)^{-1} a_{0}$ as we did previously and use equivariance to write $I(t)$ as follows:

$$
\left\langle\mathcal{K}(c(t), a(t)), \frac{\delta l}{\delta \xi}(\xi(t), a(t))\right\rangle=\left\langle\mathcal{K}\left(c_{0}, a_{0}\right), g(t)\left[\frac{\delta l}{\delta \xi}(\xi(t), a(t))\right]\right\rangle
$$

The $g^{-1}$ pulls over to the right side as $g$ (and not $g^{-1}$ ) because of our conventions of always using left representations. We now differentiate the right hand side of this equation. To do so, we use the following well known formula for differentiating the coadjoint action (see Marsden and Ratiu [1994], page 276):

$$
\frac{d}{d t}[g(t) \mu(t)]=g(t)\left[-\operatorname{ad}_{\xi(t)}^{*} \mu(t)+\frac{d}{d t} \mu(t)\right],
$$

where, as usual,

$$
\xi(t)=g(t)^{-1} \dot{g}(t) .
$$

Using this and the Euler-Poincaré equations, we get

$$
\begin{aligned}
\frac{d}{d t} I & =\frac{d}{d t}\left\langle\mathcal{K}\left(c_{0}, a_{0}\right), g(t)\left[\frac{\delta l}{\delta \xi}(\xi(t), a(t))\right]\right\rangle \\
& =\left\langle\mathcal{K}\left(c_{0}, a_{0}\right), \frac{d}{d t}\left\{g(t)\left[\frac{\delta l}{\delta \xi}(\xi(t), a(t))\right]\right\}\right\rangle \\
& =\left\langle\mathcal{K}\left(c_{0}, a_{0}\right), g(t)\left[-\operatorname{ad}_{\xi}^{*} \frac{\delta l}{\delta \xi}+\operatorname{ad}_{\xi}^{*} \frac{\delta l}{\delta \xi}+\frac{\delta l}{\delta a} \diamond a\right]\right\rangle \\
& =\left\langle\mathcal{K}\left(c_{0}, a_{0}\right), g(t)\left[\frac{\delta l}{\delta a} \diamond a\right]\right\rangle \\
& =\left\langle g(t)^{-1} \mathcal{K}\left(c_{0}, a_{0}\right),\left[\frac{\delta l}{\delta a} \diamond a\right]\right\rangle \\
& =\left\langle\mathcal{K}(c(t), a(t)),\left[\frac{\delta l}{\delta a} \diamond a\right]\right\rangle
\end{aligned}
$$

where, in the last steps we used the definitions of the coadjoint action, the EulerPoincaré equation (3.4) and the equivariance of the map $\mathcal{K}$.

Corollary 4.2 For the pure Euler-Poincaré equations, the Kelvin quantity $I(t)$, defined the same way as above but with $I: \mathcal{C} \times \mathfrak{g} \rightarrow \mathbb{R}$, is conserved.

The Heavy Top. As a simple illustration of the results so far, we consider the heavy top. For continuum examples, we refer to Holm, Marsden and Ratiu [1997] for fluid theories and to Cendra, Holm, Hoyle and Marsden [1997] for Vlasov plasmas.

The heavy top kinetic energy is given by the left invariant metric on $S O(3)$ whose value at the identity is $\langle\mathbf{x}, \mathbf{y}\rangle=\mathbb{I} \mathbf{x} \cdot \mathbf{y}$, where $\mathbf{x}, \mathbf{y} \in \mathbb{R}^{3}$ are thought of as elements of $\mathfrak{s o}(3)$, the Lie algebra of $S O(3)$, via the isomorphism $\mathbf{x} \in \mathbb{R}^{3} \mapsto \hat{\mathbf{x}} \in \mathfrak{s o}(3)$, $\hat{\mathbf{x}} \mathbf{y}:=\mathbf{x} \times \mathbf{y}$, and where $\mathbb{I}$ is the (time independent) moment of inertia tensor in body 
coordinates, usually taken as a diagonal matrix by choosing the body coordinate system to be a principal axes body frame. This kinetic energy is thus left invariant under the full group $S O(3)$. The potential energy is given by the work done in lifting the weight of the body to the height of its center of mass, with the direction of gravity pointing downwards. If $m$ denotes the total mass of the top, $g$ the magnitude of the gravitational acceleration, $\chi$ the unit vector of the oriented line segment pointing from the fixed point about which the top rotates (the origin of a spatial coordinate system) to the center of mass of the body, and $l$ its length, then the potential energy is given by $-m g l \mathbf{R}^{-1} \mathbf{e}_{3} \cdot \boldsymbol{\chi}$, where $\mathbf{e}_{3}$ is the axis of the spatial coordinate system parallel to the direction of gravity but pointing upwards and where $\mathbf{R} \in \mathrm{SO}(3)$ is the orientation of the body. This potential energy breaks the full $S O(3)$ symmetry and is invariant only under the rotations $S^{1}$ about the $\mathbf{e}_{3}$-axis.

To apply Theorem 3.1, we take the Lagrangian of the heavy top to be the kinetic minus the potential energy, regarded as a function on $T S O(3) \times \mathbb{R}^{3} \rightarrow \mathbb{R}$. In particular, the potential is given by $U\left(u_{\mathbf{R}}, \mathbf{v}\right)=m g l \mathbf{R}^{-1} \mathbf{v} \cdot \boldsymbol{\chi}$, where $u_{\mathbf{R}} \in T_{\mathbf{R}} \mathrm{SO}(3)$ (eventually identified with $\dot{\mathbf{R}}$ ) and $\mathbf{v} \in \mathbb{R}^{3}$ (eventually identified with the direction of gravity), which is easily seen to be $S O(3)$-invariant. Thus, the heavy top equations of motion in the body representation are given by the Euler-Poincaré equations (3.4) for the Lagrangian $l: \mathfrak{s o}(3) \times \mathbb{R}^{3} \rightarrow \mathbb{R}$. To compute the explicit expression of $l$, denote by $\boldsymbol{\Omega}$ the angular velocity and by $\boldsymbol{\Pi}=\mathbb{I} \boldsymbol{\Omega}$ the angular momentum in the body representation. Let $\boldsymbol{\Gamma}=\mathbf{R}^{-1} \mathbf{v}$; if $\mathbf{v}=\mathbf{e}_{3}$, the unit vector pointing upwards on the vertical spatial axis, then $\boldsymbol{\Gamma}$ is this unit vector viewed by an observer fixed and moving with the body. The Lagrangian $l: \mathfrak{s o}(3) \times \mathbb{R}^{3} \rightarrow \mathbb{R}$ is thus given by

$$
l(\boldsymbol{\Omega}, \boldsymbol{\Gamma})=L\left(\mathbf{R}^{-1} u_{\mathbf{R}}, \mathbf{R}^{-1} \mathbf{v}\right)=\frac{1}{2} \boldsymbol{\Pi} \cdot \boldsymbol{\Omega}+m g l \boldsymbol{\Gamma} \cdot \boldsymbol{\chi} .
$$

It is now straightforward to compute the Euler-Poincaré equations. First note that

$$
\frac{\delta l}{\delta \boldsymbol{\Omega}}=\boldsymbol{\Pi}, \quad \frac{\delta l}{\delta \boldsymbol{\Gamma}}=m g l \boldsymbol{\chi}
$$

Since

$$
\operatorname{ad}_{\boldsymbol{\Omega}}^{*} \boldsymbol{\Pi}=\boldsymbol{\Pi} \times \boldsymbol{\Omega}, \quad \mathbf{v} \diamond \boldsymbol{\Gamma}=-\boldsymbol{\Gamma} \times \mathbf{v}, \text { and } \quad \hat{\Omega} \boldsymbol{\Gamma}=-\boldsymbol{\Gamma} \times \Omega,
$$

the Euler-Poincaré equations are

$$
\dot{\boldsymbol{\Pi}}=\boldsymbol{\Pi} \times \boldsymbol{\Omega}+m g l \boldsymbol{\Gamma} \times \chi
$$

which are coupled to the $\boldsymbol{\Gamma}$ evolution $\dot{\boldsymbol{\Gamma}}=\boldsymbol{\Gamma} \times \boldsymbol{\Omega}$. This system of two vector equations for $\Pi, \Gamma$ are the classical Euler-Poisson equations and they describe the motion of the heavy top in the body representation.

To illustrate the Kelvin-Noether theorem, choose $\mathcal{C}=\mathfrak{g}$ and let $\mathcal{K}: \mathcal{C} \times V^{*} \rightarrow$ $\mathfrak{g}^{* *} \cong \mathfrak{g}$ be the map $(W, \boldsymbol{\Gamma}) \mapsto W$. Then the Kelvin-Noether theorem gives the statement

$$
\frac{d}{d t}\langle W, \Pi\rangle=m g l\langle W, \boldsymbol{\Gamma} \times \chi\rangle
$$

where $W(t)=\mathbf{R}(t)^{-1} \mathbf{w}$; in other words, $W(t)$ is the body representation of a space fixed vector. This statement is easily verified directly. Also, note that 
$\langle W, \Pi\rangle=\langle\mathbf{w}, \pi\rangle$, with $\boldsymbol{\pi}=\mathbf{R}(t) \boldsymbol{\Pi}$, so the Kelvin-Noether theorem may be viewed as a statement about the rate of change of the momentum map of the heavy top (that is, the spatial angular momentum) relative to the full group of rotations, not just those about the vertical axis.

\section{General Lagrangian Reduction.}

The Lagrangian analogue of cotangent bundle reduction was developed by Cendra, Ibort and Marsden [1987] and Marsden and Scheurle [1993a,b]. (Routh, already around 1860 investigated in coordinates what we would call today the abelian version.) These references developed the coordinate version of the resulting reduced Euler-Lagrange equations as well as the corresponding associated reduction of variational principles. Another interesting paper in this subject is that of Weinstein [1996] who used groupoids to show, amongst other things, one can regard discrete and continuous Lagrangian reduction from a single viewpoint. Discrete Lagrangian systems with symmetry, following ideas of Veselov and Moser are of great interest in numerical algorithms (see Wendlandt and Marsden [1997] and references therein),

One starts with a $G$-invariant Lagrangian $L$ on $T Q$, which induces a Lagrangian $l$ on the quotient space $(T Q) / G$, An important point, but which is easy to see, is that, assuming the group actions are free and proper, this quotient space $(T Q) / G$ is intrinsically a bundle over $T(Q / G)$ with a fiber modeled on the Lie algebra $\mathfrak{g}$. Denote, in a local trivialization, the variables in the base, or shape space $Q / G$ by $r^{\alpha}, \dot{r}^{\alpha}$ and the fiber variables by $\xi^{\alpha}$. In such a local trivialization, the reduced equations are the Hamel equations:

$$
\begin{aligned}
\frac{d}{d t} \frac{\partial l}{\partial \dot{r}^{\alpha}}-\frac{\partial l}{\partial r^{\alpha}} & =0 \\
\frac{d}{d t} \frac{\partial l}{\partial \xi^{b}}-\frac{\partial l}{\partial \xi^{a}} C_{d b}^{a} \xi^{d} & =0 .
\end{aligned}
$$

However, for applications to stability problems as well as being global, it is desirable to choose a connection $\mathcal{A}$, say the mechanical connection, on the bundle $Q \rightarrow Q / G$. Then the variables in the shape space and the vertical part $\Omega$ of a velocity vector on $Q$ become globally and intrinsically defined. In coordinates, the vertical part is given by $\Omega^{a}=\xi^{a}+A_{\alpha}^{a}(r) \dot{r}^{\alpha}$ where the components of the connection with respect to the local coordinates are denoted $A_{\alpha}^{a}$. In such variables, the resulting reduced Euler-Lagrange equations are

$$
\begin{aligned}
\frac{d}{d t} \frac{\partial l}{\partial \dot{r}^{\alpha}}-\frac{\partial l}{\partial r^{\alpha}} & =\frac{\partial l}{\partial \Omega^{a}}\left(-B_{\alpha \beta}^{a} \dot{r}^{\beta}+\mathcal{E}_{\left.\alpha d^{a} \Omega^{d}\right)}\right. \\
\frac{d}{d t} \frac{\partial l}{\partial \Omega^{b}} & =\frac{\partial l}{\partial \Omega^{a}}\left(-\mathcal{E}_{\alpha \beta}^{a} \dot{r}^{\alpha}+C_{d b}^{a} \Omega^{d}\right)
\end{aligned}
$$

where $B_{\alpha \beta}^{a}$ is the curvature of the connection $\mathcal{A}_{\alpha}^{b}, C_{b d}^{a}$ are the structure constants of the Lie algebra $\mathfrak{g}$ and where $\mathcal{E}_{\alpha d}^{a}=C_{b d}^{a} \mathcal{A}_{\alpha}^{b}$. This second set of equations are generalizations of the Euler-Poincaré equations. The two sets of equations are called, respectively, the horizontal and vertical equations. 
This theory has had a large impact on, for example, the theory of nonholonomic systems (see Bloch, Krishnaprasad, Marsden and Murray [1996]). It has also proven very useful in optimal control problems. For example, it was used in Koon and Marsden [1997a] to extend the falling cat theorem of Montgomery [1990] to the case of nonholonomic systems.

The work of Cendra, Marsden and Ratiu [1997] develops the geometry and variational structure of the reduced Euler-Lagrange equations. This work starts with the following view of the bundle picture. As above, choose a connection $\mathcal{A}$ on $Q \rightarrow Q / G$ such as the mechanical connection and let $\tilde{\mathfrak{g}}$ denote the vector bundle over shape space $Q / G$ which is the associated bundle to $\mathfrak{g}$ and the adjoint action of $G$ on $\mathfrak{g}$. There is a bundle isomorphism

$$
T Q / G \cong T(Q / G) \oplus \tilde{\mathfrak{g}}
$$

determined by the projection on the first factor and by the vertical projection associated with the connection on the second. The sum is a Whitney sum (i.e., fiberwise a direct sum) of vector bundles over $Q / G$.

Using the geometry of this bundle $T Q / G=T(Q / G) \oplus \tilde{\mathfrak{g}}$, one obtains a nice intrinsic interpretation of the above reduced Euler-Lagrange equations in terms of covariant derivatives of induced connections. One easily gets the dynamics of particles in a Yang-Mills fields as well as many other interesting examples (such as a rigid body with rotors, etc.) as special cases. We will illustrate this with Wong's equations below. Preliminary calculations show that in nonholonomic mechanics we will get a beautiful geometric interpretation of the important momentum equation of Bloch, Krishnaprasad, Marsden and Murray [1996].

The dual bundle picture. The above construction sheds light on the bundle picture for cotangent bundles mentioned earlier. Taking the dual of the above isomorphism gives an isomorphism

$$
T^{*} Q / G \cong T^{*}(Q / G) \oplus \tilde{\mathfrak{g}}^{*}
$$

Like any quotient Poisson manifold, the space $\left(T^{*} Q\right) / G$ has a natural Poisson structure. The description of this Poisson structure viewed on the bundle $T^{*}(Q / G) \oplus \tilde{\mathfrak{g}}^{*}$ involves a synthesis of the canonical bracket, the Lie-Poisson bracket, and curvature and is a very interesting way of encoding the original description of Montgomery, Marsden and Ratiu [1984] of the Poisson structure on $\left(T^{*} Q\right) / G$. One gets from this picture both the usual Lie-Poisson description of dynamics and the Hamiltonian description of semidirect product theory.

Reduced variational principles. The above picture also leads naturally to a generalization of the reduced variational principles described for the Euler-Poincaré equations above. One does this by dividing the variations in Hamilton's principle on $T^{*} Q$ into horizontal and vertical parts, which then drop appropriately to the quotient space in a manner similar to that in the Euler-Poincaré case. 
We regard this reduction of variational principles as fundamental. Another approach to reduction of Lagrangian systems is based on reducing geometric objects such as almost tangent structures (see for example, De Leon, Mello and Rodrigues [1992] and subsequent papers of this group). The present framework seems to further clarify this, and identifies exactly which part of the reduced space is a tangent bundle and which part is of Euler-Poincaré type.

Stability under reduction. One of the motivations is to produce a category, a Lagrangian analogue of the symplectic and Poisson category, that is stable under reduction. As the Euler-Poincaré equations show, this category cannot be that of tangent bundles or even locally tangent bundle-like objects.

However, the above ideas lead to an answer to this question in a natural way. We enlarge the traditional category for Lagrangian mechanics, namely tangent bundles, to bundles of the form

$$
T Q \oplus U
$$

where $U$ is a vector bundle with a connection over $Q$, each fiber of which carries a Lie algebra structure and where the base $Q$ also carries a two form (a "magnetic term"). This structure is rich enough to include the reduced Euler-Lagrange equations (by taking $U=\tilde{\mathfrak{g}}$ ) and carry variational methods.

In addition, this category is stable under reduction. If one reduces this structure by the action of a group $K$, then $Q$ gets replaced by $Q / K$, while the fiber expands by enlarging each Lie algebra fiber by a group extension construction, with the base two form playing the role of the cocycle. This provides a Lagrangian analogue of the reduction by stages procedure of Marsden, Misiolek, Perlmutter and Ratiu [1997].

Wong's Equations. As another illustration of the ideas of Lagrangian reduction, we consider the important example of Wong's equations. See Montgomery [1984] and the references therein for the original papers. This example is important for its own sake, but also through the fact that it enters into some fundamental optimal control problems, as shown by Montgomery [1990] and Koon and Marsden [1997a].

We begin with a Riemannian manifold $Q$ with a free and proper isometric action of a Lie group $G$ on $Q$. Let $\mathcal{A}$ denote the mechanical connection; that is, it is the principal connection whose horizontal space is the metric orthogonal to the group orbits. The quotient space $Q / G=X$ inherits a Riemannian metric from that on $Q$. Given a curve $c(t)$ in $Q$, we shall denote the corresponding curve in the base space $X$ by $r(t)$.

The optimal control problem under consideration is as follows:

Isoholonomic Problem (Falling Cat Problem). Fixing two points $q_{1}, q_{2} \in Q$, among all curves $q(t) \in Q, 0 \leq t \leq 1$ such that $q(0)=q_{0}, q(1)=q_{1}$ and $\dot{q}(t) \in \operatorname{hor}_{q(t)}$ (horizontal with respect to the mechanical connection $\mathcal{A}$ ), find the curve or curves $q(t)$ such that the energy of the base space curve, namely,

$$
\frac{1}{2} \int_{0}^{1}\|\dot{r}\|^{2} d t,
$$


is minimized.

Theorem 5.1 (Montgomery $(\mathbf{1 9 9 0 , 1 9 9 1 ) . ) ~ I f ~} q(t)$ is a (regular) optimal trajectory for the isoholonomic problem, then there exists a curve $\lambda(t) \in \mathfrak{g}^{*}$ such that the reduced curve $r(t)$ in $X=Q / G$ together with $\lambda(t)$ satisfies Wong's equations:

$$
\begin{aligned}
\dot{p}_{\alpha} & =-\lambda_{a} \mathcal{B}_{\alpha \beta}^{a} \dot{r}^{\beta}-\frac{1}{2} \frac{\partial g^{\beta \gamma}}{\partial r^{\alpha}} p_{\beta} p_{\gamma} \\
\dot{\lambda}_{b} & =-\lambda_{a} C_{d b}^{a} \mathcal{A}_{\alpha}^{d} \dot{r}^{\alpha}
\end{aligned}
$$

where $g_{\alpha \beta}$ is the local representation of the metric on the base space $X$; that is

$$
\frac{1}{2}\|\dot{r}\|^{2}=\frac{1}{2} g_{\alpha \beta} \dot{r}^{\alpha} \dot{r}^{\beta}
$$

$g^{\beta \gamma}$ is the inverse of the matrix $g_{\alpha \beta}, p_{\alpha}$ is defined by

$$
p_{\alpha}=\frac{\partial l}{\partial \dot{r}^{\alpha}}=g_{\alpha \beta} \dot{r}^{\beta},
$$

and where we write the components of $\mathcal{A}$ as $\mathcal{A}_{\alpha}^{b}$ and similarly for its curvature $\mathcal{B}$.

Proof. By general principles in the calculus of variations, given an optimal solution $q(t)$, there is a Lagrange multiplier $\lambda(t)$ such that the new action function defined on the space of curves with fixed endpoints by

$$
\mathfrak{S}[q(\cdot)]=\int_{0}^{1}\left[\frac{1}{2}\|\dot{r}(t)\|^{2}+\langle\lambda(t), \mathcal{A} \dot{q}(t)\rangle\right] d t
$$

has a critical point at this curve. Using the integrand as a Lagrangian, identifying $\Omega=\mathcal{A} \dot{q}$ and applying the reduced Euler-Lagrange equations to the reduced Lagrangian

$$
l(r, \dot{r}, \Omega)=\frac{1}{2}\|\dot{r}\|^{2}+\langle\lambda, \Omega\rangle
$$

then gives Wong's equations by the following simple calculations:

$$
\frac{\partial l}{\partial \dot{r}^{\alpha}}=g_{\alpha \beta} \dot{r}^{\beta} ; \quad \frac{\partial l}{\partial r^{\alpha}}=\frac{1}{2} \frac{\partial g^{\beta \gamma}}{\partial r^{\alpha}} \dot{r}^{\beta} \dot{r}^{\gamma} ; \quad \frac{\partial l}{\partial \Omega^{a}}=\lambda_{a} .
$$

The constraints are $\Omega=0$ and so the reduced Euler- Lagrange equations become

$$
\begin{aligned}
\frac{d}{d t} \frac{\partial l}{\partial \dot{r}^{\alpha}}-\frac{\partial l}{\partial r^{\alpha}} & =-\lambda_{a}\left(\mathcal{B}_{\alpha \beta}^{a} \dot{r}^{\beta}\right) \\
\frac{d}{d t} \lambda_{b} & =-\lambda_{a}\left(\mathcal{E}_{\alpha b}^{a} \dot{r}^{\alpha}\right)=-\lambda_{a} C_{d b}^{a} \mathcal{A}_{\alpha}^{d} \dot{r}^{\alpha} .
\end{aligned}
$$

But

$$
\begin{aligned}
\frac{d}{d t} \frac{\partial l}{\partial \dot{r}^{\alpha}}-\frac{\partial l}{\partial r^{\alpha}} & =\dot{p}_{\alpha}-\frac{1}{2} \frac{\partial g_{\beta \gamma}}{\partial r^{\alpha}} \dot{r}^{\beta} \dot{r}^{\gamma} \\
& =\dot{p}_{\alpha}+\frac{1}{2} \frac{\partial g^{\kappa \sigma}}{\partial r^{\alpha}} g_{\kappa \beta} g_{\sigma \gamma} \dot{r}^{\beta} \dot{r}^{\gamma} \\
& =\dot{p}_{\alpha}+\frac{1}{2} \frac{\partial g^{\beta \gamma}}{\partial r^{\alpha}} p_{\beta} p_{\gamma},
\end{aligned}
$$


and so we have the desired equations.

Let us now give another version of this procedure in abstract notation and, at the same time, show how it fits in with the bundle view of Lagrangian reduction. Again, let $\pi: Q \rightarrow X$ be a principal bundle with structure group $G$, a Lie group acting on the left, let $\mathcal{A}$ be a principal connection on $Q$ and let $\mathcal{B}$ be the curvature of $\mathcal{A}$. Suppose that $g$ is a given Riemannian metric on $X$ and let $\nabla$ be the corresponding Levi-Civita connection. Assume, for simplicity, that $G$ is a compact group with a bi-invariant Riemannian metric $K$.

Define the Lagrangian $L: T Q \rightarrow \mathbb{R}$ by

$$
L(q, \dot{q})=\frac{1}{2} K(A(q, \dot{q}), A(q, \dot{q}))+\frac{1}{2} g(\pi(q))(T \pi(q, \dot{q}), T \pi(q, \dot{q})) .
$$

An element of $\tilde{\mathfrak{g}}$ has the form $\bar{v}=[q, v]_{G}$ where $q \in Q$ and $v \in \mathfrak{g}$ and where $[q, v]_{G}$ denotes the equivalence class of the pair $(q, v)$ with respect to the group action by $G$. Since $K$ is bi-invariant, its restriction to $\mathfrak{g}$ is Ad-invariant, and therefore we can define $K\left([q, v]_{G},[q, v]_{G}\right)=K(v, v)$.

The reduced bundle is

$$
T(Q / G) \oplus \tilde{\mathfrak{g}} \equiv T X \oplus \tilde{\mathfrak{g}}
$$

and a typical element of it is denoted $(x, \dot{x}, \bar{v})$. The reduced Lagrangian is given by

$$
l(x, \dot{x}, \bar{v})=\frac{1}{2} K(v, v)+\frac{1}{2} g(x)(x, \dot{x}) .
$$

Now we will write the vertical and horizontal reduced Euler-Lagrange equations. An arbitrary variation $\delta \bar{v}$ in the direction of the fiber of $\tilde{\mathfrak{g}}$ is of type $\delta \bar{v}=[q, \delta v]_{G}$, where $\delta v \in \mathfrak{g}$ is arbitrary. We have

$$
\frac{\partial l}{\partial \bar{v}}(x, \dot{x}, \bar{v}) \delta \bar{v}=K(v, \delta v)
$$

and

$$
\left(\operatorname{ad}_{\bar{v}}^{*} \frac{\partial l}{\partial \bar{v}}(x, \dot{x}, \bar{v})\right) \delta \bar{v}=K(v,[v, \delta v]) .
$$

Since $K$ is bi-invariant, we have $K\left(\operatorname{ad}_{w} u, v\right)+K\left(u, \operatorname{ad}_{w} v\right)=0$, for all $u, v$, and $w$ in $\mathfrak{g}$. Therefore, $K(v,[v, \delta v])=-K([v, v], \delta v)=0$. Thus the vertical reduced Euler-Lagrange equation is one of Wong's equations

$$
\frac{d}{d t} K(v, \cdot)=0
$$

This actually agrees with the previous form of Wong's equations since $\lambda$ represents the value of the body momentum while the form $K(v, \cdot)$ represents the spatial momentum, which is conserved. After some straightforward calculations, the horizontal reduced Euler-Lagrange equation becomes in this case

$$
-\left(\nabla_{\dot{\gamma}} \dot{\gamma}\right)^{b}=K(v, \tilde{\mathcal{B}}(x)(\dot{x}, \cdot)),
$$

which is the other of Wong's equations. Here $\tilde{\mathcal{B}}$ denotes the curvature thought of as a Lie algebra valued two form on the base. 


\section{The Euler-Poincaré Equations via Lagrangian Reduc- tion}

Now we are ready to explain how the Euler-Poincaré equations can be derived using the techniques of Lagrangian reduction. This should be plausible from what we have already said simply because the coordinate expression for the reduced EulerLagrange equations include a generalized form of Euler-Poincaré equations. But we need to make this connection precise.

The set up. We consider a slight generalization of the Euler-Poincaré equations given earlier. This time we consider a group $G$ acting on a vector space $V$ and hence on its dual $V^{*}$. We also consider a configuration space $Q$, but in this section, $G$ will act trivially on $Q$. Consider a Lagrangian

$$
L: T(G \times Q) \times V^{*} \rightarrow \mathbb{R}
$$

where $G$ is a group, $Q$ is a manifold and $V^{*}$ is the dual of the vector space $V$. The value of $L$ at the point $\left(g, q, \dot{g}, \dot{q}, a_{0}\right) \in T(G \times Q) \times V^{*}$ will be denoted $L\left(g, q, \dot{g}, \dot{q}, a_{0}\right)$, as usual, and, as in the discussion of the Euler-Poincaré equations earlier, we think of $a_{0}$ as being a parameter that remains fixed along the evolution of the system.

By construction, the action of $G$ on $V$ and $V^{*}$ satisfies the property

$$
\langle g a, g v\rangle=\langle a, v\rangle
$$

for all $a \in V^{*}$, all $v \in V$ and all $g \in G$. (We will often write $\langle a, v\rangle=\langle v, a\rangle$ ).

Assume that $L$ has the following invariance property:

$$
L\left(g^{\prime} g, q, g^{\prime} \dot{g}, \dot{q}, g^{\prime} a_{0}\right)=L\left(g, q, \dot{g}, \dot{q}, a_{0}\right)
$$

for all $a_{0} \in V^{*}$, all $q \in Q$ and all $g^{\prime}, g \in G$. Let

$$
L(e, q, \xi, \dot{q}, a)=l(\xi, q, \dot{q}, a),
$$

for all $\xi \in \mathfrak{g}$, all $q \in Q$ and all $a \in V^{*}$. Then the invariance property implies, for all $g \in G$, all $q \in Q$ and all $a \in V^{*}$,

$$
L\left(g, q, \dot{g}, \dot{q}, a_{0}\right)=l(\xi, q, \dot{q}, a)
$$

where $\xi=g^{-1} \dot{g}$ and $a=g^{-1} a_{0}$.

A small generalization of the argument given earlier for the Euler-Poincaré equations proves the next result.

Theorem 6.1 The following conditions are equivalent:

(i) The curve $\left(g(t), q(t), a_{0}\right)$ is a critical point of the action

$$
\int_{t_{0}}^{t_{1}} L\left(g, q, \dot{g}, \dot{q}, a_{0}\right) d t
$$

with restrictions on variations given by $\delta g\left(t_{i}\right)=0$ for $i=0,1, \delta q\left(t_{i}\right)=0$ for $i=0,1$, and $\delta a_{0}=0$. 
(ii) The curve $(\xi(t), q(t), a(t))$, where $a(t)=g^{-1}(t) a_{0}$ for all $t$ and $\xi(t)=g^{-1}(t) \dot{g}$, is a critical point of the action

$$
\int_{t_{0}}^{t_{1}} l(\xi, q, \dot{q}, a) d t
$$

with restrictions on variations given by

$$
\delta \xi=\dot{\eta}+[\xi, \eta]
$$

where $\eta$ is any curve on $\mathfrak{g}$ such that $\eta\left(t_{i}\right)=0$ for $i=0,1$,

$$
\delta q\left(t_{i}\right)=0
$$

for $i=0,1$ and

$$
\delta a=-\eta a
$$

and, besides, the curve $a(t)$ must satisfy

$$
\dot{a}+\xi a=0
$$

for all t. (As earlier, this last condition comes from the condition $\dot{a}_{0}=0$ together with $a_{0}=g a$.)

A direct application of $(i i)$ leads to the reduced equations

$$
\frac{d}{d t} \frac{\delta l}{\delta \xi}=\operatorname{ad}_{\xi}^{*} \frac{\delta l}{\delta \xi}+\frac{\delta l}{\delta a} \diamond a
$$

together with the standard Euler-Lagrange equations for $q$ :

$$
\frac{\partial l}{\partial q}-\frac{d}{d t} \frac{\partial l}{\partial \dot{q}}=0
$$

Recall that, by definition,

$$
\langle v \diamond a, \zeta\rangle=-\langle\zeta a, v\rangle
$$

for all $\zeta \in \mathfrak{g}$, all $a \in V^{*}$ and all $v \in V$. The first of these equations is of course the Euler-Poincaré equation. These and the Euler-Lagrange equations for $q$, together with the equation $\dot{a}+\xi a=0$ form a complete set of equations of the system in terms of the variables $(\xi, q, a)$. This framework is applied to Vlasov plasmas in Cendra, Holm, Hoyle and Marsden [1997].

Now we shall recast conditions $(i)$ and $(i i)$ into an equivalent form. The idea is to introduce the condition that $a_{0}$ is conserved by making it the momentum conjugate to a cyclic variable, just as one does for the charge in Kaluza-Klein theory. Thus, let us define the Lagrangian

$$
\bar{L}: T\left(G \times Q \times V^{*} \times V\right) \rightarrow \mathbb{R}
$$

by

$$
\bar{L}\left(g, q, a_{0}, v_{0}, \dot{g}, \dot{q}, \dot{a}_{0}, \dot{v}_{0}\right)=L\left(g, q, \dot{g}, \dot{q}, a_{0}\right)+\left\langle a_{0}, \dot{v}_{0}\right\rangle
$$


Notice that for the Lagrangian $\bar{L}, a_{0}$ is the momentum conjugate to the cyclic variable $v_{0}$, and hence is a constant. The variable $v_{0}$ is not constant, but its evolution is the first order equation $\dot{v}_{0}+\partial L / \partial a_{0}=0$. Thus, the Euler-Lagrange equations for $\bar{L}$ are equivalent to the Euler-Lagrange equations for $L$ with the parameter $a_{0}$ fixed (together with the equation for $v_{0}$ ).

Thus, the strategy is to perform reduction of $L$ by the equivalent process of performing standard tangent bundle Lagrangian reduction of $\bar{L}$.

Now we observe that

$$
G \times Q \times V^{*} \times V \rightarrow\left(G \times Q \times V^{*} \times V\right) / G
$$

is a principal bundle with structure group $G$ acting as before, that is, $g^{\prime}\left(g, q, a_{0}, v_{0}\right)=$ $\left(g^{\prime} g, q, g^{\prime} a_{0}, g^{\prime} v_{0}\right)$. Moreover, this bundle is isomorphic, as a principal bundle, to the trivial bundle

$$
G \times Q \times V^{*} \times V \rightarrow Q \times V^{*} \times V
$$

where the action of $G$ is given by $g^{\prime} \cdot(g, q, a, v)=\left(g^{\prime} g, q, a, v\right)$, for all $g^{\prime}, g \in G$, all $a \in V^{*}$ and all $v \in V$. This assertion follows using the isomorphism $\psi: G \times Q \times$ $V^{*} \times V \rightarrow G \times Q \times V^{*} \times V$ given by $\psi\left(g, q, a_{0}, v_{0}\right)=\left(g, q, g^{-1} a_{0}, g^{-1} v_{0}\right) \equiv(g, q, a, v)$. It is easy to check that $\psi\left(g^{\prime}\left(g, q, a_{0}, v_{0}\right)\right)=g^{\prime} \cdot \psi\left(g, q, a_{0}, v_{0}\right) \equiv g^{\prime} \cdot(g, q, a, v)$, for all $g^{\prime}, g \in G$, all $a_{0} \in V^{*}$ and all $v_{0} \in V$. One also checks that the composition $\bar{L} \circ T \psi^{-1}=: L^{V}$ is given by

$$
L^{V}(g, q, a, v, \dot{g}, \dot{q}, \dot{a}, \dot{v})=L(g, q, \dot{g}, \dot{q}, a)+\left\langle a, \dot{v}+g^{-1} \dot{g} v\right\rangle .
$$

¿From now on we will use the trivial bundle described above and the Lagrangian $L^{V}$.

Remark. Using techniques like those in Cendra and Marsden [1987], which give a version of the Lagrange multiplier theorem, we can show that conditions $(i)$ and (ii) are equivalent to either of the following conditions,

(iii) The curve $\left(g(t), q(t), a_{0}, v_{0}\right)$ is a critical point of the action

$$
\int_{t_{0}}^{t_{1}} \bar{L}\left(g, q, a_{0}, v_{0}, \dot{g}, \dot{q}, \dot{a}_{0}, \dot{v}_{0}\right) d t
$$

for variations satisfying the endpoint conditions $\delta g\left(t_{i}\right)=0, \delta q\left(t_{i}\right)=0$, $\delta a_{0}\left(t_{i}\right)=0$ and $\delta v_{0}\left(t_{i}\right)=0$ for $i=0,1$.

(iv) The curve $(g(t), q(t), a(t), v(t))$ is a critical point of the action

$$
\int_{t_{0}}^{t_{1}} L^{V}(g, q, a, v, \dot{g}, \dot{q}, \dot{a}, \dot{v}) d t
$$

for variations satisfying the endpoint conditions $\delta g\left(t_{i}\right)=0, \delta q\left(t_{i}\right)=0, \delta a\left(t_{i}\right)=$ 0 and $\delta v\left(t_{i}\right)=0$ for $i=0,1$. 
We shall show that the reduced Euler-Lagrange equations for the Lagrangian $L^{V}$ are the same as the reduced system for $l$. To do this, we first calculate the reduced bundle $T\left(Q \times V^{*} \times V\right) \oplus \tilde{\mathfrak{g}}$. Since the bundle $G \times Q \times V^{*} \times V$ is trivial, the bundle $\tilde{\mathfrak{g}}$ is $\tilde{\mathfrak{g}} \equiv Q \times V^{*} \times V \times \mathfrak{g}$. The Lie algebra structure on $\tilde{\mathfrak{g}}$ is given by $\left[\left(q, a, v, \xi_{1}\right),\left(q, a, v, \xi_{2}\right)\right]=\left(q, a, v,\left[\xi_{1}, \xi_{2}\right]\right)$.

Let us choose the trivial principal connection $\mathcal{A}$ on $G \times Q \times V^{*} \times V$, that is, the connection given by $\mathcal{A}(g, q, a, v, \dot{g}, \dot{q}, \dot{a}, \dot{v})=\dot{g} g^{-1}$. Since the connection is trivial, it has zero curvature. The reduced Lagrangian is clearly given by

$$
l^{V}(\xi, q, a, v, \dot{q}, \dot{a}, \dot{v})=l(\xi, q, \dot{q}, a)+\langle a, \dot{v}+\xi v\rangle .
$$

Now one is ready to calculate the reduced Euler-Lagrange equations for $l^{V}$ using this set up. This can be done in coordinates using the coordinate version of the reduced Euler-Lagrange equations we gave earlier, or in their intrinsic formulation.

Since the connection is trivial and its curvature is zero, the horizontal equations for $l^{V}$ are simply the usual Euler-Lagrange equations for these variables; these give the equations for $q$ as well as the equations for $a$ and $v$. The Euler-Lagrange equation for $q$ with respect to $l^{V}$ is clearly the same as the Euler-Lagrange equation for $q$ with respect to $l$. The horizontal equation for $a$ is the equation

$$
\frac{d}{d t} \frac{\partial l^{V}}{\partial \dot{a}}-\frac{\partial l^{V}}{\partial a}=0
$$

which, since $l^{V}$ does not depend on $\dot{a}$, is simply

$$
\dot{v}+\xi v+\frac{\partial l}{\partial a}=0
$$

which is equivalent to the equation $\dot{v}_{0}+\partial L / \partial a_{0}=0$ that we had before.

The Euler-Lagrange equation for $v$ is

$$
\frac{d}{d t} \frac{\partial l^{V}}{\partial \dot{v}}-\frac{\partial l^{V}}{\partial v}=0
$$

Since $\partial l^{V} / \partial \dot{v}=a$ and $\partial l^{V} / \partial v=-\xi a$, this gives the correct equation $\dot{a}+\xi a=0$ for $a$.

The vertical equation becomes

$$
\frac{d}{d t} \frac{\partial l^{V}}{\partial \xi}=\operatorname{ad}_{\xi}^{*}\left(\frac{\partial l^{V}}{\partial \xi}\right) .
$$

Clearly

$$
\frac{\partial l^{V}}{\partial \xi}=\frac{\partial l}{\partial \xi}+v \diamond a
$$

and so the vertical equation becomes

$$
\frac{d}{d t} \frac{\partial l}{\partial \xi}+\dot{v} \diamond a+v \diamond \dot{a}=\operatorname{ad}_{\xi}^{*} \frac{\partial l}{\partial \xi}+\operatorname{ad}_{\xi}^{*}(v \diamond a) .
$$


Using the equations for $\dot{a}$ and $\dot{v}$, this becomes

$$
\frac{d}{d t} \frac{\partial l}{\partial \xi}-\left(\xi v+\frac{\partial l}{\partial a}\right) \diamond a-v \diamond(\xi a)=\operatorname{ad}_{\xi}^{*} \frac{\partial l}{\partial \xi}+\operatorname{ad}_{\xi}^{*}(v \diamond a) .
$$

i.e.,

$$
\frac{d}{d t} \frac{\partial l}{\partial \xi}-\operatorname{ad}_{\xi}^{*} \frac{\partial l}{\partial \xi}-\frac{\partial l}{\partial a} \diamond a=(\xi v) \diamond a+v \diamond(\xi a)+\operatorname{ad}_{\xi}^{*}(v \diamond a) .
$$

But the right hand side is identically zero, as is seen from the definitions. Thus, we have achieved our goal of recovering the Euler-Poincaré equations with an advected parameter from Lagrangian reduction.

\section{References}

Abarbanel, H.D.I., D.D. Holm, J.E. Marsden, and T.S. Ratiu [1986] Nonlinear stability analysis of stratified fluid equilibria, Phil. Trans. Roy. Soc. London A 318, 349-409; also Richardson number criterion for the nonlinear stability of three-dimensional stratified flow, Phys. Rev. Lett. 52 [1984], 2552-2555.

Abraham, R. and J.E. Marsden [1978] Foundations of Mechanics, Second Edition, Addison-Wesley.

Arnold, V.I. [1966a] Sur la géometrie differentielle des groupes de Lie de dimenson infinie et ses applications à l'hydrodynamique des fluids parfaits, Ann. Inst. Fourier, Grenoble 16, 319-361.

Arnold, V.I. [1966b] On an a priori estimate in the theory of hydrodynamical stability, Izv. Vyssh. Uchebn. Zaved. Mat. Nauk 54, 3-5; English Translation: Amer. Math. Soc. Transl. 79 [1969], 267-269.

Arnold, V.I. [1966c] Sur un principe variationnel pour les découlements stationaires des liquides parfaits et ses applications aux problemes de stabilité non linéaires, J. Mécanique 5, 29-43.

Arnold, V.I. (ed.) [1988] Dynamical Systems III, Encyclopedia of Mathematics 3, Springer-Verlag.

Arnold, V.I. and B. Khesin [1992] Topological methods in hydrodynamics, Ann. Rev. Fluid Mech. 24, 145-166.

Arnold, V.I. and B. Khesin [1997] Topological methods in Fluid Dynamics, Appl. Math. Sciences, Springer-Verlag.

Bloch, A.M., P.S. Krishnaprasad, J.E. Marsden, and R. Murray [1996] Nonholonomic mechanical systems with symmetry, Arch. Rat. Mech. An., 136, 21-99.

Bloch, A.M., P.S. Krishnaprasad, J.E. Marsden, and T.S. Ratiu [1994] Dissipation induced instabilities, Ann. Inst. H. Poincaré, Analyse Nonlineare 11, 37-90. 
Bloch, A.M., P.S. Krishnaprasad, J.E. Marsden, and T.S. Ratiu [1996] The EulerPoincaré equations and double bracket dissipation, Comm. Math. Phys. 175, $1-42$.

Cendra, H. and J.E. Marsden [1987] Lin constraints, Clebsch potentials and variational principles, Physica D 27, 63-89.

Cendra, H., A. Ibort, and J.E. Marsden [1987] Variational principal fiber bundles: a geometric theory of Clebsch potentials and Lin constraints, J. Geom. Phys. 4, 183-206.

Cendra, H., D.D. Holm, M.J.W. Hoyle and J. E. Marsden [1997] The MaxwellVlasov equations in Euler-Poincaré form, preprint.

Cendra, H., J. E. Marsden and T.S. Ratiu [1997] Lagrangian reduction by stages, preprint.

Chetayev, N.G. [1941] On the equations of Poincaré, J. Appl. Math. Mech. 5, $253-262$

De Leon, M. M. H. Mello, and P.R. Rodrigues [1992] Reduction of nondegenerate nonautonomous Lagrangians. Cont. Math. AMS 132, 275-306.

Ebin, D.G. and J.E. Marsden [1970] Groups of diffeomorphisms and the motion of an incompressible fluid, Ann. Math. 92, 102-163.

Guichardet, A. [1984] On rotation and vibration motions of molecules, Ann. Inst. H. Poincaré 40, 329-342.

Guillemin, V. and S. Sternberg [1980] The moment map and collective motion, Ann. of Phys. 1278, 220-253.

Guillemin, V. and S. Sternberg [1984] Symplectic Techniques in Physics, Cambridge University Press.

Hamel, G [1904] Die Lagrange-Eulerschen Gleichungen der Mechanik, Z. für Mathematik u. Physik 50, 1-57.

Hamel, G [1949] Theoretische Mechanik, Springer-Verlag.

Holm, D.D. [1996] Hamiltonian balance equations, Physica D, 98 (1996) 379-414.

Holm, D.D. and B.A. Kupershmidt [1983] Poisson brackets and Clebsch representations for magnetohydrodynamics, multifluid plasmas, and elasticity, Physica D 6, 347-363.

Holm, D.D., J.E. Marsden, and T.S. Ratiu [1986] The Hamiltonian structure of continuum mechanics in material, spatial and convective representations, Séminaire de Mathématiques supérie, Les Presses de L'Univ. de Montréal 100, 11-122. 
Holm, D. D., Marsden, J. E. and Ratiu, T. [1997] The Euler-Poincaré equations and semidirect products with applications to continuum theories, preprint.

Holm, D.D., J.E. Marsden, T.S. Ratiu, and A. Weinstein [1985] Nonlinear stability of fluid and plasma equilibria, Phys. Rep. 123, 1-116.

Holmes, P.J. and J.E. Marsden [1983] Horseshoes and Arnold diffusion for Hamiltonian systems on Lie groups, Indiana Univ. Math. J. 32, 273-310.

Iwai, T. [1987] A geometric setting for classical molecular dynamics, Ann. Inst. Henri Poincaré, Phys. Th. 47, 199-219.

Koon, W.S. and J.E. Marsden [1997a] Optimal control for holonomic and nonholonomic mechanical systems with symmetry and Lagrangian reduction, SIAM J. Control and Optim. 35, 901-929.

Kummer, M. [1981] On the construction of the reduced phase space of a Hamiltonian system with symmetry, Indiana Univ. Math. J. 30, 281-291.

Kupershmidt, B.A. and T. Ratiu [1983] Canonical maps between semidirect products with applications to elasticity and superfluids, Comm. Math. Phys. 90, $235-250$.

Lagrange, J.L. [1788] Mëcanique Analitique, Chez la Veuve Desaint

Leonard, N.E. and J.E. Marsden [1997] Stability and Drift of Underwater Vehicle Dynamics: Mechanical Systems with Rigid Motion Symmetry, Physica D 105, 130-162.

Lie, S. [1890] Theorie der Transformationsgruppen, Zweiter Abschnitt, Teubner, Leipzig.

Marsden, J.E. [1982] A group theoretic approach to the equations of plasma physics, Can. Math. Bull. 25, 129-142.

Marsden, J.E., G. Misiolek, M. Perlmutter and T.S. Ratiu [1997] Reduction by stages and group extensions, in preparation.

Marsden, J.E., G.W. Patrick and S. Shkoller [1997] Variational methods in continuous and discrete mechanics and field theory, preprint.

Marsden, J.E. and T.S. Ratiu [1994] Introduction to Mechanics and Symmetry, Texts in Applied Mathematics, 17, Springer-Verlag.

Marsden, J.E., T.S. Ratiu, and A. Weinstein [1984a] Semi-direct products and reduction in mechanics, Trans. Am. Math. Soc. 281, 147-177.

Marsden, J.E., T.S. Ratiu, and A. Weinstein [1984b] Reduction and Hamiltonian structures on duals of semidirect product Lie Algebras, Cont. Math. AMS 28, 55-100. 
Marsden, J.E. and J. Scheurle [1993a] Lagrangian reduction and the double spherical pendulum, ZAMP 44, 17-43.

Marsden, J.E. and J. Scheurle [1993b] The reduced Euler-Lagrange equations, Fields Institute Comm. 1, 139-164.

Marsden, J.E. and A. Weinstein [1974] Reduction of symplectic manifolds with symmetry, Rep. Math. Phys. 5, 121-130.

Marsden, J.E., A. Weinstein, T.S. Ratiu, R. Schmid, and R.G. Spencer [1983] Hamiltonian systems with symmetry, coadjoint orbits and plasma physics, in Proc. IUTAM-IS1MM Symposium on Modern Developments in Analytical Mechanics, Torino 1982, Atti della Acad. della Sc. di Torino 117, 289-340.

Montgomery, R. [1984] Canonical formulations of a particle in a Yang-Mills field, Lett. Math. Phys. 8, 59-67.

Montgomery, R. [1986] The bundle picture in mechanics, Ph.D. Thesis, Berkeley.

Montgomery, R. [1990] Isoholonomic problems and some applications, Comm. Math Phys. 128, 565-592.

Montgomery, R. [1991] Optimal control of deformable bodies and its relation to gauge theory, in The Geometry of Hamiltonian Systems, T. Ratiu ed., Springer-Verlag.

Montgomery, R., J.E. Marsden, and T.S. Ratiu [1984] Gauged Lie-Poisson structures, Cont. Math. AMS 28, 101-114.

Ovsienko, V.Y. and B.A. Khesin [1987] Korteweg-de Vries superequations as an Euler equation, Funct. Anal. and Appl. 21, 329-331.

Poincaré, H. [1901] Sur la stabilité de l'équilibre des figures piriformes affectées par une masse fluide en rotation, Philosophical Transactions A 198, 333-373.

Poincaré, H. [1910] Sur la precession des corps deformables, Bull Astron 27, 321356.

Ratiu. T.S. [1980a] The Euler-Poissot equations and integrability Thesis, University of California at Berkeley.

Ratiu. T.S. [1981] Euler-Poisson equations on Lie algebras and the $N$-dimensional heavy rigid body, Proc. Natl. Acad. Sci. USA 78, 1327-1328.

Ratiu, T.S. [1982] Euler-Poisson equations on Lie algebras and the $N$-dimensional heavy rigid body, Am. J. Math. 104, 409-448, 1337.

Satzer, W.J. [1977] Canonical reduction of mechanical systems invariant under abelian group actions with an application to celestial mechanics, Ind. Univ. Math. J. 26, 951-976. 
Smale, S. [1970] Topology and mechanics, Inv. Math. 10, 305-331, 11, 45-64.

Sudarshan, E.C.G. and N. Mukunda [1974] Classical Mechanics: A Modern Perspective, Wiley, New York, 1974; Second Edition, Krieber, Melbourne-Florida, 1983.

Vinogradov, A.M. and B.A. Kupershmidt [1977] The structures of Hamiltonian mechanics, Russ. Math. Surv. 32, 177-243.

Weinstein, A. [1996] Lagrangian Mechanics and Groupoids Fields Inst. Comm., 7, $207-231$.

Wendlandt, J.M. and J.E. Marsden [1997] Mechanical integrators derived from a discrete variational principle, Physica D 106, 223-246. 